\title{
Correction to: Impulsive Control Problems Without the Frobenius Condition
}

\section{Correction to:}

Chapter 4 in: A. Arutyunov et al., Optimal Impulsive

Control, Lecture Notes in Control and Information

Sciences 477, https://doi.org/10.1007/978-3-030-02260-0_4

In the original version of the book, the following belated correction has been incorporated in Chapter 4. In Theorem 4.1, the condition (b) has been changed from 'The set $U$ and cone $K$ are convex.' to 'The set $f(x, U, t)$ and cone $K$ are convex.' The correction chapter and the book have been updated with the change. 